difficulties. We decided that a temporary aortic transection approach would provide better surgical exposure and enable manipulation of the tumor, which we hoped would shorten the cardiac arrest time. Although our procedure resulted in incomplete excision, we achieved our primary purpose of preventing cardiopulmonary collapse as a result of tumor compression. This case demonstrates that a temporary aortic transection approach may be justified for appropriate patients who otherwise would have no viable treatment option.

\section{References}

1. Ducatman BS, Scheithauter MD, Piepgras DG, Reiman HM, Ilstrup DM. Malignant peripheral nerve sheath tumor: a clinicopathologic study of 120 cases. Cancer. 1986;57:2006-21.

2. Reynolds RM, Browning GGP, Nawroz I, Campbell IW. Von Recklinghausen's neurofibromatosis: neurofibromatosis type 1. Lancet. 2003;361:1552-4.

3. Vaporciyan AA, Rice D, Correa AM, Walsh G, Putnam JB, Swisher S, et al. Resection of advanced thoracic malignancies requiring cardiopulmonary bypass. Eur J Cardiothorac Surg. 2002;22:47-52.

\title{
Twisting of pedicled left internal thoracic artery graft three hundred sixty degrees clockwise: Does it change the outcome?
}

\author{
Apurba Kumar Sarma, MCh, Sai Kiran Krishnamurthy Venkata Sathya Siva, \\ MS, Jayakumar Karunakaran, MCh, and Neelakandhan Kurur Sankaran, MCh, \\ Thiruvananthapuram, India
}

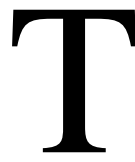

wisting of aorta-coronary venous conduits has been reported previously with fatal outcome and is an extremely uncommon occurrence. ${ }^{1}$ However, twisting of the pedicled left internal thoracic artery (LITA) graft was not found to be reported in a search of the English-language literature (MEDLINE and PUBMED), and its outcome is unknown. We report a case of an inadvertent clockwise $360^{\circ}$ twisting of a LITA graft sequentially anastomosed to the diagonal and left anterior descending coronary arteries (LAD) with an angiographically documented patency with good distal runoff.

\section{Clinical Summary}

A 54-year-old man had a history of class III unstable angina. Echocardiographic evaluation was unremarkable. The treadmill test result was positive for inducible ischemia. Coronary angiography showed a normal left main coronary artery and total occlusion of the proximal LAD. The diagonal artery was arising from the diseased segment of the LAD. The left circumflex artery was nondominant and normal. The right coronary artery was totally

\footnotetext{
From the Department of Cardio-vascular and Thoracic Surgery, Sree Chitra Tirunal Institute for Medical Sciences and Technology, Thiruvananthapuram, India.

Received for publication Jan 16, 2004; accepted for publication Jan 27, 2004.

Address for reprints: Apurba K. Sarma, MCh, Flat No. B2, New Faculty Building, SCT Housing Colony, Kumarapuram, Poonthi Road, Thiruvananthapuram, 695011 Kerala, India (E-mail: aks@ sctimst.ac.in).

J Thorac Cardiovasc Surg 2004;128:617-8

$0022-5223 / \$ 30.00$

Copyright $\odot 2004$ by The American Association for Thoracic Surgery

doi:10.1016/j.jtcvs.2004.01.025
}

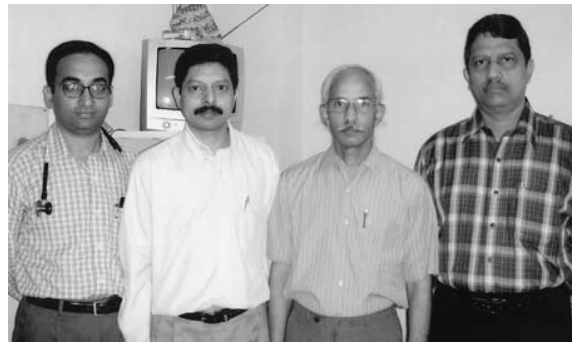

Sai Kiran, Sarma, Neelakandhan, Jayakumar (left to right)

occluded past the right ventricular branch. A left ventricular angiogram showed good function, and there was no regional wall motion abnormality or mitral regurgitation.

Coronary artery bypass grafting was performed by sequential grafting of the LITA to the diagonal artery and the LAD and a reversed saphenous vein graft to the distal right coronary artery under conventional cardiopulmonary bypass. After the distal anastomoses were completed, just before removal of the vascular clip from the LITA, we realized that the pedicle was twisted $360^{\circ}$ clockwise proximal to the LITA-diagonal artery anastomosis. Although distal runoff in the diagonal and LAD was good, we were concerned about the patency of the graft.

The following options were contemplated: (1) to leave it as it was, (2) to transect the LITA at the origin and make it a free graft after untwisting, and (3) to revise the distal anastomoses. The third option was not considered further because of the involved technical difficulties. We selected the first option. The patient was weaned from cardiopulmonary bypass on the first attempt without any inotropic support and was shifted to the intensive care unit for elective ventilation. The next morning, after extubation, coronary angiography was repeated and showed a patent LITA conduit with good flow to the diagonal artery and the LAD (Figure 1). Postoperative electrocardiography revealed a normal pattern with no ST-T changes. Predischarge echocardiography did not show any regional wall motion abnormality. The patient was discharged on the sixth postoperative day. At 3 months of follow-up, he has resumed all his physical activities and is free from angina. Coronary angiography repeated at 6 months showed a patent LITA graft with good flow to the LAD and the diagonal artery (Figure 2).

\section{Discussion}

The frequency of twisting of coronary bypass conduit is unknown. Roberts and colleagues ${ }^{1}$ reported twisting of venous bypass conduits. All 3 affected patients died, 2 patients on the operating table 

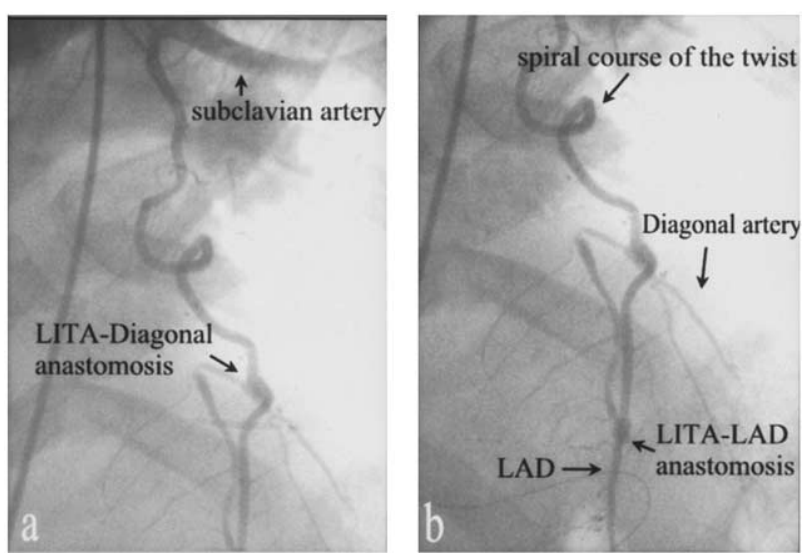

Figure 1. Anteroposterior view with $35^{\circ}$ cranial tilt showing $360^{\circ}$ twist as spiral loop of patent LITA conduit with flow to diagonal and LAD.

and 1 about 6 hours after entering the recovery room from the operating room. In an experimental study, Endean and associates ${ }^{2}$ showed thrombosis of venous grafts within 4 hours at $200^{\circ}$ twist and further reported that a slight amount of graft twist probably does not reduce flow. They also concluded that twisting of more than $135^{\circ}$ greatly reduces flow, leading to early graft thrombosis.

We accepted the twist in the LITA conduit for several reasons. There was good distal runoff. Myocardial activity returned soon after removal of the vascular clip from the LITA. The heart picked up in sinus rhythm after the release of aortic crossclamp. Hemodynamic values were normal. Multilead electrocardiography was normal. The patient could be weaned from cardiopulmonary bypass on the first attempt without any inotropic support.

This patient's LITA conduit was long; therefore the twist can be perceived as a loop in an anteroposterior view with a $35^{\circ}$ cranial tilt (Figure 1). We believe that because of the long length of the
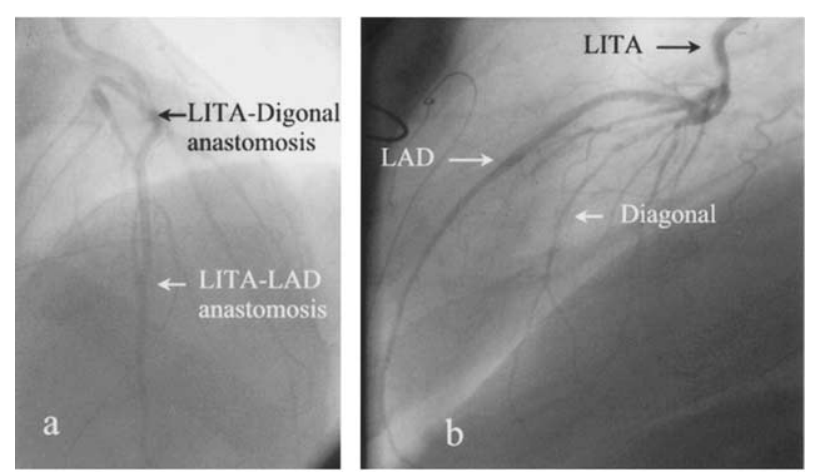

Figure 2. Repeat coronary angiogram at 6 months of follow-up showing patent LITA conduit and distal runoff.

conduit the twist acquired a spiral course, forming a loop, rather than being limited to a short segment, which would otherwise have compromised the flow. Also, in a pedicled conduit the effect of the twist may not be transmitted to the LITA because of the thickness of the pedicle. Further, the architecture of the arterial wall and the absence of valves may play a part in keeping the lumen patent even when there is a significant twist.

Although our experience is limited to this single case, we conclude that even a significant twist in the pedicled arterial conduit is unlikely to produce compromise of the flow to the target vessels so long as there is good distal runoff.

\section{References}

1. Roberts WC, Lachman AS, Virmani R. Twisting of an aorta-coronary bypass conduit: a complication of coronary surgery. $J$ Thorac Cardiovasc Surg. 1978;75:772-6.

2. Endean ED, DeJong S, Dobrin PB. Effect of twist on flow and patency of vein grafts. J Vasc Surg. 1989;9:651-5. 